\title{
Pseudopotential Study of CdTe Quantum Dots: Electronic and Optical Properties
}

\author{
Fadila Mezrag ${ }^{a}$, Nadir Bouarissa ${ }^{a}$ *(1) \\ ${ }^{a}$ Laboratory of Materials Physics and Its Applications, University of M'sila, 28000 M'sila, Algeria
}

Received: December 27, 2017; Revised: April 11, 2019; Accepted: April 29, 2019

\begin{abstract}
The present contribution reports on the electronic and optical properties of zinc-blende CdTe quantum dots using a pseudopotential approach. Our findings showed that the predicted nano-structured direct band gap is significantly increased relative to the bulk one. For a nanocrystal diameter in the range 1.2-5 nm, the refractive index and dielectric constant are found to be highly reduced relative the bulk values. Beyond a nanocrystal diameter of $5 \mathrm{~nm}$, the size dependence of all features of interest becomes weak and tends towards retrieving the bulk value.
\end{abstract}

Keywords: Electronic properties, Optical constants, Quantum dots, CdTe, Pseudopotentials.

\section{Introduction}

CdTe is a semiconductor compound made from cadmium (Cd) and tellurium (Te). It is a stable crystalline direct bandgap material. It is used for optical windows and lenses as an infrared optical material. It is also applied for electro-opticmodulator. Usually, the material is sandwiched with CdS in order to form a p-n junction of solar cells. Besides, it is used to make thin film solar cells. Across a wide range of temperatures, $\mathrm{CdTe}$ is proven to give a good performance. At room temperature, the material of interest allows the construction of compact detectors that have many applications in nuclear spectroscopy ${ }^{1}$.

Quantum dots (QDs) are very small semiconductor particles ${ }^{2}$. Many types of them, when exposed to light or applied electricity will emit light of specific frequencies which can be precisely tuned by varying the shape, size and material of the QDs. This gives rise to the change of QDs properties as a function of size, shape and material ${ }^{3}$. As a matter of fact, QDs exhibit intermediate properties between those of discrete molecules, and those of bulk semiconductors. Due to the highly tunable properties of QDs, these materials are of wide interest and found potential applications which include light emitting diodes, diode lasers, transistors, quantum computing, solar cells and medical imaging ${ }^{4}$.

In the present contribution, the electronic energy levels and optical properties of CdTe spherical quantum dots are investigated. By reducing the size of CdTe crystals to a few nanometers or less, CdTe QDs can be obtained. The objective of this paper is to show how the properties of CdTe spherical QDs vary by changing the QDs size. The calculations are essentially based on the empirical pseudopotential method (EPM). This approach even at the nanoscale has proven to give good results ${ }^{5-8}$.

\section{Computational Method}

The calculations are performed using essentially the EPM $^{9,10}$. For bulk CdTe, the pseudopotential Hamiltonian for an electron in the crystal is written as,

$$
H=-\left(\hbar^{2} / 2 m\right) \nabla^{2}+V(r)
$$

The potential $V(r)$ which depends on the position $r$ is then expanded in reciprocal lattice vectors by using Fourier transform. The band structure is obtained by diagonalizing the Hamiltonian $\mathrm{H}$ on a small plane wave basis set.

The Schrödinger equation is solved by determining the roots of the secular equation which are derived from the Hamiltonian matrix.

The pseudopotential form factors are obtained by fitting the atomic form factors to experiment. The adjustment is made using a non-linear least-squares fitting procedure ${ }^{11,12}$. In the present contribution, the experimental and previous theoretical band-gap energies for bulk CdTe at the highsymmetry points $\Gamma, \mathrm{X}$ and L fixed in the fits are $1.5045 \mathrm{eV}^{13}$, $3.48^{14}$, and $2.47 \mathrm{eV}^{14}$, respectively. The final adjusted pseudopotential form factors of bulk CdTe are determined as: $V_{S}(3)=-0.396388, V_{S}(8)=0.198275, V_{S}(11)=0.459548$, $V_{A}(3)=0.1311, V_{A}(4)=0.28$, and $V_{A}(11)=0.708145$ Ry.

For investigating QDs, the application of the EPM requires the reduction of the dimensionality of the environment of electrons to a zero-dimension. This results in discretization of energy states giving rise to energy levels instead of band structure ${ }^{15}$. By imposing proper boundary conditions on the wave functions, which are dependent on the size and shape of the dots, i.e.,

$$
k=\frac{\pi}{\sqrt{3 a}}\left(n_{x}, n_{y}, n_{z}\right)=\frac{\pi}{\sqrt{3 a}} n
$$


Where $\mathrm{a}$ is the spherical QD radius, the energy levels have been determined.

The quantum confinement effect on the energy band gap $E_{g}$ of CdTe QDs is obtained using the expression ${ }^{16,17}$,

$$
E_{g}=E_{g}^{0}+\frac{\hbar^{2} \pi^{2}}{2 a^{2}}\left[\frac{1}{m_{e}^{*}}+\frac{1}{m_{h}^{*}}\right]-\frac{1.8 e^{2}}{\varepsilon a}
$$

where $E_{g}^{0}$ is the CdTe bulk band gap, $\varepsilon$ is the effective dielectric constant, and $m_{e}^{*}$ and $m_{h}^{*}$ are the electron and heavy hole effective masses for bulk CdTe, respectively which are calculated using the same approach as that used by Bouarissa ${ }^{18}$.

\section{Results and Discussion}

Figure 1 shows the size dependence of the direct $(\Gamma-\Gamma)$ band-gap energy for zinc-blende CdTe quantum dots with a spherical shape. The quantum dot radius is taken in the range 1.2-5 $\mathrm{nm}$. We observe that the band gap energy $(\Gamma-\Gamma)$ increases rapidly as far as the quantum dot radius is reduced. Qualitatively the same behavior has been reported for nanostructured $\mathrm{GaAs}{ }^{19}$ and $\mathrm{GaN}$ and InAs spherical quantum dots ${ }^{8,20}$. Thus, one can expect that beyond a size of $5 \mathrm{~nm}$, the decrease of the direct $(\Gamma-\Gamma)$ band-gap energy becomes weak and tends towards retrieving the bulk value. In fact, as far as the size of the quantum dots is reduced, the quantum confinement effect becomes more important, i.e. much bigger than that in the case of CdTe quantum dots with larger sizes. In semiconductors, the Coulomb interaction between electrons and holes has important effects on their optical properties, especially close to the absorption edge. The electrons and holes can pair together and form excitons. By analogy to the hydrogen atom, the exciton has a Bohr radius. The exciton radius is much larger than the Bohr radius of the hydrogen atom and depends highly on the semiconductor under consideration. In our case, the exciton Bohr radius is known to be $1.05 \mathrm{~nm}$ for CdTe material. As a matter of fact, when the size of the quantum dot is much

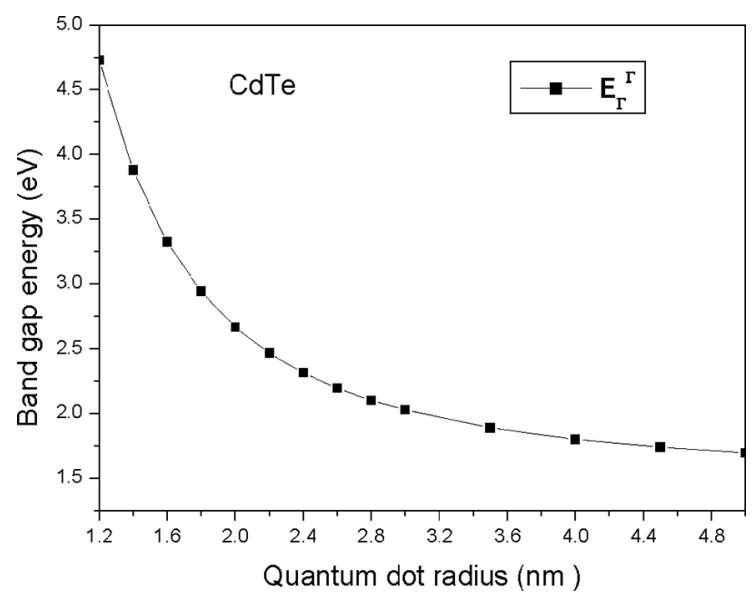

Figure 1. Direct band-gap $(\Gamma-\Gamma)$ in nanosized CdTe as a function of quantum dot radius. larger than the exciton Bohr radius, the effect of the dielectric confinement becomes weak and consequently the situation becomes similar to that in the bulk CdTe. This is consistent with our results. Moreover, in the present contribution, the bulk direct $(\Gamma-\Gamma)$ band-gap energy is found to be $1.50 \mathrm{eV}$. This value is in perfect accord with the experimental one reported in Ref. ${ }^{13}$ for bulk CdTe at room temperature.

The refractive index (n) of semiconductors is an important physical parameter which describes their optical properties ${ }^{21,22}$. Moreover, the knowledge of $\mathrm{n}$ is a useful information for the fabrication of devices such as wave guides, photonic crystals and solar cells, to name a few. This is true for both bulk and nanostructured materials. In the present paper $n$ has been calculated for zinc-blend CdTe quantum dots with a spherical shape as a function of quantum dot size in the range 1.2-5 nm using five different models as reported in Ref. ${ }^{23}$. The models are: (i) the Moss formula ${ }^{24}$ as revised by Ravindra and Srivastava ${ }^{25}$. (ii) the Gupta and Ravindra ${ }^{26}$ relation (iii) the Hervé and Vandamme empirical expression ${ }^{27}$ (iv) the Reddy and Anjaneyulu ${ }^{28}$ relation and (v) the Reddy and Ahammed empirical relation ${ }^{29}$. Our obtained results are displayed in Figure 2. Note that for all models of interest, $\mathrm{n}$ for CdTe quantum dots is decreased quickly as the size of the quantum dot is reduced. The behavior seems to be monotonic. The experimental value of $\mathrm{n}$ for the bulk CdTe is reported to be $2.72^{30}$. The best accord with our obtained values calculated from the above mentioned models is obtained when the model of Moss ${ }^{24}$ is used, where $n$ is found to be 2.91. This value deviates from the experimental one of 2.72 by less than $7 \%$ and is in perfect accord with that of 2.91 calculated by Hannachi and Bouarissa ${ }^{31}$. With a nanocrystal diameter in the range $1.2-5 \mathrm{~nm}, \mathrm{n}$ appears to be importantly reduced relative to the bulk value especially when approaching a size of $1.2 \mathrm{~nm}$. This is true for all used models of interest. The present data of $n$ obtained from the model of Moss are fitted by a least-squares procedure. As a result, we obtain the following analytical relation expressing thus $\mathrm{n}$ as a function of the quantum dots radius $\mathrm{a}$,

$$
n(a)=1.70+0.52 a-0.06 a^{2}
$$

Another interesting physical parameter is the dielectric constant. The latter measures the ability of a substance to insulate charges from each other. Its accurate determination is very useful for the design and fabrication of wave guiding devices ${ }^{32,33}$. In this work the high-frequency dielectric constant $\left(\varepsilon_{\infty}\right)$ has been calculated using the relation,

$$
\varepsilon_{\infty}=n^{2}
$$

Here $\mathrm{n}$ is the refractive index calculated from the model of Moss which has been shown to give the best accord with experiment as regards $\mathrm{n}$ for $\mathrm{CdTe}$. The value of $\varepsilon_{\infty}$ calculated in the present work for bulk CdTe is found to be 8.46 . The 
discrepancy between this value and that of 8.47 reported by Hannachi and Bouarissa ${ }^{31}$ is less than $1 \%$.

The variation in $\varepsilon_{\infty}$ as a function of the CdTe quantum dot radius is displayed in Figure 3. We observe that when the size of CdTe quantum dot is increased, $\varepsilon_{\infty}$ increases as well, exhibiting a non-linear and monotonic behavior. Thus, one expects that the value of $\varepsilon_{\infty}$ for CdTe quantum dots are smaller than that of bulk CdTe. As a matter of fact, semiconductor materials with high dielectric constants are useful in the manufacture of high-value capacitors and become important mainly in memory cell dielectrics, passive components and gate dielectrics. The fit of our $\varepsilon_{\infty}$ data using a least-squares procedure has given the dependence of $\varepsilon_{\infty}$ on the quantum dot radius a as follows,

$$
\varepsilon_{\infty}(a)=2.36+2.51 a-0.28 a^{2}
$$

Accordingly, we note that $\varepsilon_{\infty}$ is non-linearly dependent on the CdTe quantum dot radius.

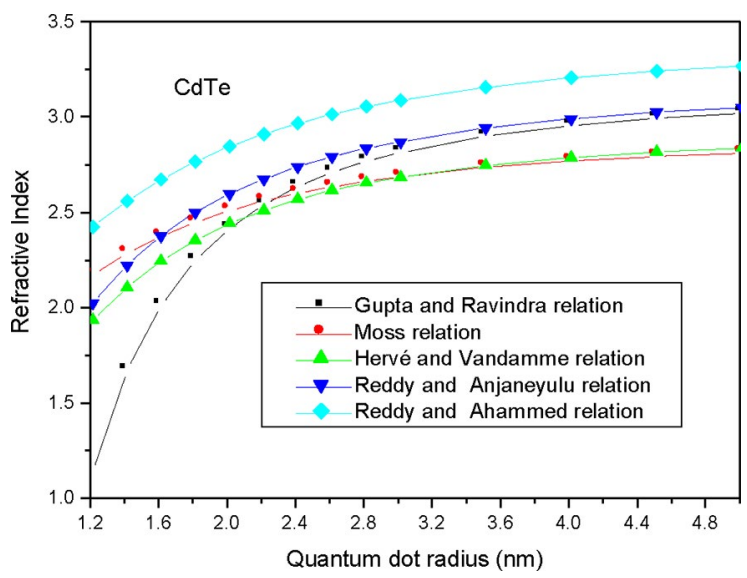

Figure 2. Refractive index in nanosized CdTe as a function of quantum dot radius using various models.

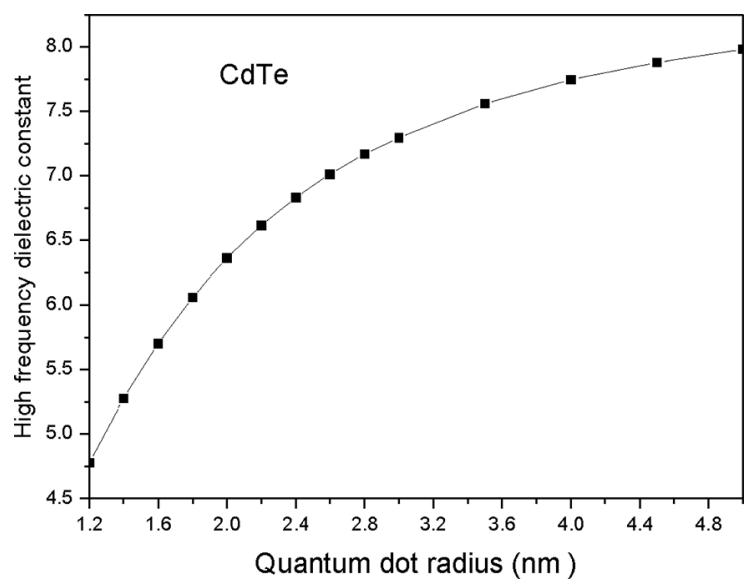

Figure 3. High-frequency dielectric constant in nanosized CdTe as a function of quantum dot radius using Ravindra model.

\section{Conclusion}

The dependence of the direct $(\Gamma-\Gamma)$ energy band-gap, refractive index and high-frequency dielectric constant on the nanocrystal size of zinc-blende CdTe quantum dots with spherical shape was investigated. The quantum dot radius was taken in the range $1.2-5 \mathrm{~nm}$. The calculations were mainly performed using a pseudopotential approach. The refractive index was calculated using various models. The Moss model was found to give better accord with experiment than the other models of interest. Our findings for bulk CdTe were found to be in good accord with experiment and previous theoretical data available in the literature. Within a nanocrystal diameter being considered here, the values of the refractive index and dielectric constant were found to be highly reduced relative to the bulk values. This has been attributed to the confinement effect. Polynomials were provided so as to approximate the refractive index and high-frequency dielectric constant as a function of the nanocrystal size.

\section{References}

1. Capper P, ed. Properties of Narrow Gap Cadmium-based Compounds. London: INSPEC; 1994.

2. Ashoori RC. Electrons in artificial atoms. Nature. 1996;379:413-419.

3. Murray CB, Kagan CR, Bawendi MG. Synthesis and Characterization of Monodisperse Nanocrystals and Close-Packed Nanocrystal Assemblies. Annual Review of Materials Science. 2000;30:545-610.

4. Ramírez HY, Flórez J, Camacho AS. Efficient control of coulomb enhanced second harmonic generation from excitonic transitions in quantum dot ensembles. Physical Chemistry Chemical Physics. 2015;17(37):23938-23946.

5. Bester G. Electronic excitations in nanostructures: an empirical pseudopotential based approach. Journal of Physics: Condensed Matter. 2009;21(2):023202.

6. Kent PRC, Bellaiche L, Zunger A. Pseudopotential theory of dilute III-V nitrides. Semiconductor Science and Technology. 2002;17(8):851-859.

7. Bouarissa N. Phonon Confinement in Nanostructured InP. Journal of Computational and Theoretical Nanoscience. 2013;10(5):1284-1289.

8. Mezrag F, Bouarissa N, Boucenna M. The size-dependent electronic and optical properties of InAs quantum dots. Optik. 2016;127(3):1167-1170.

9. Cohen ML, Chelikowsky JR. Electronic Structure and Optical Properties of Semiconductors. Berlin: Springer; 1989.

10. Bouarissa N. Electronic properties of GaxIn1-xP from pseudopotential calculations. Materials Chemistry and Physics. 2010;124(1):336-341. 
11. Kobayasi T, Nara H. Properties of Nonlocal Pseudopotentials of $\mathrm{Si}$ and Ge Optimized under Full Interdependence among Potential Parameters. Bulletin of College of Medical Sciences, Tohoku University. 1993;2(1):7-16.

12. Bouarissa N, Boucenna M. Band parameters for AlAs, InAs and their ternary mixed crystals. Physica Scripta. 2009;79(1):015701.

13. Yang J, Zidon Y, Shapira Y. Alloy composition and electronic structure of $\mathrm{Cd}_{1-\mathrm{x}} \mathrm{Zn}_{\mathrm{x}}$ Te by surface photovoltage spectroscopy. Journal of Applied Physics. 2002;91(2):703-707.

14. Kobayashi A, Sankey OF, Dow JD. Chemical trends for defect energy levels in $\mathrm{Hg}_{(1-\mathrm{x})} \mathrm{Cd}_{\mathrm{x}}$ Te. Physical Review $B$. 1982;25(10):6367-6379.

15. Kshirsagar A, Kumbhojkar N. Empirical pseudo-potential studies on electronic structure of semiconducting quantum dots. Bulletin of Materials Science. 2008;31(3):297-307.

16. Kayanuma Y. Quantum-size effects of interacting electrons and holes in semiconductor microcrystals with spherical shape. Physical Review B. 1988;38(14-15):9797-9805.

17. Ragan R. Direct energy bandgap group IV alloys and nanostructures. [Ph D. Dissertation]. Pasadena: California Institute of Technology; 2002.

18. Bouarissa N. Effective masses of electrons, heavy holes and positrons in quasi-binary $(\mathrm{GaSb})_{1-\mathrm{x}}$ (InAs) $)_{\mathrm{x}}$ crystals. Journal of Physics and Chemistry of Solids. 2006;67(7):1440-1443.

19. Al Wadiy NHM, Bouarissa N, Khan MA. Quantum confinement effects on the band structure and dielectric properties of nanostructured GaAs. Physica Scripta. 2011;84(1):015704.

20. Gueddim A, Eloud T, Messikine N, Bouarissa N. Energy levels and optical properties of GaN spherical quantum dots. Superlattices and Microstructures. 2015;77:124-133.

21. Ravindra NM, Ganapathy P, Choi J. Energy gap-refractive index relations in semiconductors - An overview. Infrared Physics \& Technology. 2007;50(1):21-29.
22. Bouarissa N. Energy gaps and refractive indices of $\mathrm{Al}_{\mathrm{x}} \mathrm{Ga}_{1-\mathrm{x}} \mathrm{As}$. Materials Chemistry and Physics. 2001;72(3):387-394.

23. Al-Assiri MS, Bouarissa N. Electronic band structure and derived properties of $\mathrm{AlAs}_{\mathrm{x}} \mathrm{Sb}_{1-\mathrm{x}}$ alloys. Superlattices and Microstructures. 2013;59:144-154.

24. Moss TS. A Relationship between the Refractive Index and the Infra-Red Threshold of Sensitivity for Photoconductors. Proceedings of the Physical Society. Section B. 1950;63(3):167174.

25. Ravindra NM, Srivastava VK. Variation of refractive index with energy gap in semiconductors. Infrared Physics. 1979;19(5):603604.

26. Gupta VP, Ravindra NM. Comments on the Moss Formula. Physica Status Solid B. 1980;100(2):715-719.

27. Hervé P, Vandamme LKJ. General relation between refractive index and energy gap in semiconductors. Infrared Physics \& Technology. 1994;35(4):609-615.

28. Reddy RR, Anjaneyulu S. Analysis of the Moss and Ravindra relations. Physica Status Solid B. 1992;174(2):K 91-K93.

29. Reddy RR, Ahammed YN. A study on the Moss relation. Infrared Physics \& Technology. 1995;36(5):825-830.

30. Palmer DW. The Semiconductors-Information Web-Site; 2008. Available from: <www.semiconductors.co.uk $>$.

31. Hannachi L, Bouarissa N. Band parameters for cadmium and zinc chalcogenide compounds. Physica B: Condensed Matter. 2009;404(20):3650-3654.

32. Adachi S. Band gaps and refractive indices of $\mathrm{AlGaAsSb}$, $\mathrm{GaInAsSb}$, and InPAsSb: Key properties for a variety of the 2-4 $\mu \mathrm{m}$ optoelectronic device applications. Journal of Applied Physics. 1987;61(10):4869-4876.

33. Bouarissa N. Pressure dependence of refractive index, dielectric constants and optical phonon frequencies of indium arsenide. Optik . 2017;138:263-268. 